\title{
Erratum to: Phenotypic and functional characterization of Glioblastoma cancer stem cells identified through 5-aminolevulinic acid-assisted surgery
}

\author{
Elena Rampazzo • Alessandro Della Puppa • \\ Chiara Frasson • Giusy Battilana $\cdot$ Sara Bianco $\cdot$ \\ Renato Scienza • Giuseppe Basso $\cdot$ Luca Persano
}

Published online: 26 August 2014

(C) Springer Science+Business Media New York 2014

\section{Erratum to: J Neurooncol (2014) 116:505-513 \\ DOI 10.1007/s11060-013-1348-3}

In the original publication, the statement in the last two sentences of the 2nd paragraph in the Discussion section should have read as follows:

"In a recent study, it has been suggested that 5-ALA does not affect the isolation and propagation of GBM initiating cells [28]. However, in our opinion, although authors compared stem cell features of $5-\mathrm{ALA}^{+}$and $5-\mathrm{ALA}^{-}$cells derived from the same tumour, their analysis is restricted to a limited number of samples [28]."

The online version of the original article can be found under doi:10. 1007/s11060-013-1348-3.

E. Rampazzo - C. Frasson - G. Battilana - S. Bianco ·

G. Basso · L. Persano $(\bowtie)$

Oncohematology Laboratory, Department of Woman and Child

Health, University of Padova, Via Giustiniani 3, 35128 Padua,

Italy

e-mail: luca.persano@unipd.it

A. Della Puppa $\cdot$ R. Scienza

Division of Neurosurgery, University-Hospital of Padova,

Via Giustiniani 2, 35128 Padua, Italy
In addition, there was an error in the title of the original publication. The word 'trough' should have read 'through'.

\section{Reference}

28. Piccirillo SG, Dietz S, Madhu B, Griffiths J, Price SJ, Collins VP, Watts C (2012) Fluorescence-guided surgical sampling of glioblastoma identifies phenotypically distinct tumour-initiating cell populations in the tumour mass and margin. $\mathrm{Br} \mathrm{J}$ Cancer 107(3):462-468 